International Research Journal of Engineering, IT \& Scientific Research
Available online at https://sloap.org/journals/index.php/irjeis/
Vol. 5 No. 5, September 2019, pages: 32 44
ISSN: 2454-2261
https://doi.org/10.21744/irjeis.v5n5.779

\title{
Research and Modeling of Wave Processes at the Scattering of Nonlinear Acoustic Waves on Cylindrical Bodies
}

Iftikhar B. Abbasov ${ }^{\text {a }}$

Article history:

Received: 27 January 2019

Accepted: 31 July 2019

Published: 30 September 2019

\section{Keywords:}

acoustic waves; cylindrical bodies; nonlinear interaction; scattering diagram; scattering;
Iftikhar B. Abbasov

2454-2261 ${ }^{\circ}$ Copyright 2019. The Author.

\section{Author correspondence:}

Iftikhar B. Abbasov,

Southern Federal University, Engineering Technological Academy,

per. Nekrasovskyi, 44, Taganrog, Russia, 347928, Russia.

Email address: iftikhar_abbasov@mail.ru

\section{Introduction}

The aquatic environment of the coastal waters of the World Ocean usually contains heterogeneities of natural and artificial origin. These heterogeneities often have a geometrically complex shape, however, bodies with a geometrically simple regular shape are first used to study them. Therefore, the problem of scattering of acoustic waves on bodies of a regular geometric shape is a standard in the waves scattering theory. Despite the sufficient elaboration of scattering problems, the question of scattering of nonlinearly interacting acoustic waves on a cylinder remains unexplored. This work is devoted to an asymptotic method for describing wave processes in the scattering of

${ }^{a}$ Southern Federal University, Rostov-on-Don, Russia 
nonlinearly interacting plane acoustic waves by cylindrical bodies and to determining the applicability limits of the proposed method (Abbasov \& Zagrai, 2019). The study of this problem can increase the efficiency of the use of hydroacoustic parametric antennas for the near location. Using a wide frequency range of secondary waves (differential, total frequency waves, and second harmonics), it is possible to obtain important information about the properties of underwater inhomogeneities.

\section{Publication Overview}

Let us consider a review of the current state of publications on this topic; the scattering problem on cylindrical bodies (or shells) is often considered for the case of falling linear acoustic waves. Therefore, we will consider publications devoted to the scattering of both linear waves and nonlinear acoustic waves. These problems are usually solved in cylindrical coordinates and have a similar geometry of the problem statement.

The case of scattering of linear acoustic waves on axisymmetric rigid bodies and shells is considered in Partridge \& Smith (1995), where the deformed cylinder method is used. The results of scattering are compared with other known methods at small angles of incidence of acoustic waves. The article Kleshchev (2011), presents the methodology and block diagram of the experiment for measuring the amplitude and phase of the acoustic field in the near zone of a cylindrical diffuser. The results of measuring the scattering characteristics of a low-frequency sound signal by elastic cylindrical shells are analyzed.

The article Andronov \& Lavrov (2015), is devoted to the study of the field in the boundary layer near the surface of an elliptical cylinder. Using the Kirchhoff formula, this asymptotics is recalculated for a narrow sector near the major axis of the cylinder section. The obtained asymptotics are uniform and allow one to follow the modification of the far-field diagram from the limiting case of the strip to the circular cylinder.

In the work (Mitri, 2016), an analytical theory using partial wave expansion is proposed for calculating acoustic backscattering by an infinitely long elliptical cylinder. By solving the system of linear equations by the matrix inversion method, the scattering coefficients for a rigid elliptic cylinder with Neumann boundary conditions are found. The results are relevant for problems of acoustic levitation and particle dynamics.

The work Gurbatov et al., (2016), is devoted to studying the influence of discrete random bottom heterogeneities on the intensity of backscattering of acoustic signals. This problem arises in the remote diagnosis of deposits of ironmanganese nodules on the seabed. The dependence of the backscattering intensity on the correlation in the mutual arrangement of particles on the bottom plane and their size distribution is considered. In the article Larin \& Tolokonikov (2015), the angular and frequency dependences of the amplitude of the scattered sound wave are calculated for a homogeneous cylinder with a thermoplastic coating. It is shown that with the help of such a coating it is possible to change the sound-reflecting properties of the cylinder. Analytical expressions are obtained for inhomogeneities of the coating material providing the lowest intensity of the scattered acoustic field.

The work Dmitriev (2018), is devoted to the study of acoustic waves scattering by inhomogeneities of small wave size using matrix Green functions. For small sizes of inhomogeneities, it is sufficient to consider the only scattering of monopole and dipole types. The results are confirmed by an analysis of the field scattered by a circular cylinder of a small wave radius in the numerical solution of the problem. The book Ostashev \& Wilson (2015), is devoted to studies of the propagation and scattering of sound in moving media with deterministic and random inhomogeneities. Modern numerical methods for calculating noise pollution in the field of atmospheric acoustics are considered.

Next, we consider several publications devoted directly to the nonlinear acoustic waves scattering by the medium inhomogeneities. An article Duck, (2002), is devoted to the study of nonlinear phenomena in the propagation of ultrasonic pulses. The problem arises in the ultrasound diagnosis of the biological environment in order to improve the visualization picture. The secondary harmonic acoustic field has a narrow spatial resolution, which is important for suppressing acoustic noise from side lobes.

The work Dos Santos et al., (2015), is devoted to the use of nonlinear wave spectroscopy for biomedical ultrasound diagnostics. Various modes of propagation of acoustic pulses in nonlinear and layered media are considered. In the work Desjouy et al., (2016), the propagation and reflection of nonlinear shock acoustic waves from surfaces with hard boundaries are studied. The results of field experiments on the reflection of acoustic waves for different angles of incidence are discussed with the results of numerical simulations based on the twodimensional Navier-Stokes equations.

Abbasov, I. (2019). A research and modeling of wave processes at the scattering of nonlinear acoustic waves on cylindrical bodies. International Research Journal of Engineering, IT \& Scientific Research, 5(5), 32-44. 


\section{Small parameter method in nonlinear acoustics}

When solving nonlinear hydrodynamic problems for specific physical conditions, a simplification of the system of equations is required. One of the methods to simplify the system of equations is the small parameter method. The small parameter expansion method $\varepsilon$ is relating to perturbation theory. In this case, the "unperturbed" problem corresponding to the zero value of the small parameter $\varepsilon=0$ is first solved. Next, by successive approximations, a solution to the "perturbed" problem is found when $\varepsilon \neq 0$. Perturbation refers to any small deviation from a simplified problem that allows an exact solution. In physics and mechanics, a limited number of problems can be solved exactly, so in almost every problem you have to use a simplified description. It comes down to finding one or more terms in the expansion of the desired solution of the small parameter. These methods belong to the class of asymptotic problems that make it possible to find out the mathematical and physical basis of the processes taking place. Their main drawback is the accuracy of the obtained asymptotic approximations; therefore, we will determine the limits of applicability of the method for our case.

A diagram of the task for diagnosing an operational assessment of the underwater situation based on a hydroacoustic parametric antenna is shown in Fig. 1. The information received from the autonomous nonlinear hydroacoustic system can be further transmitted to the following processing links.

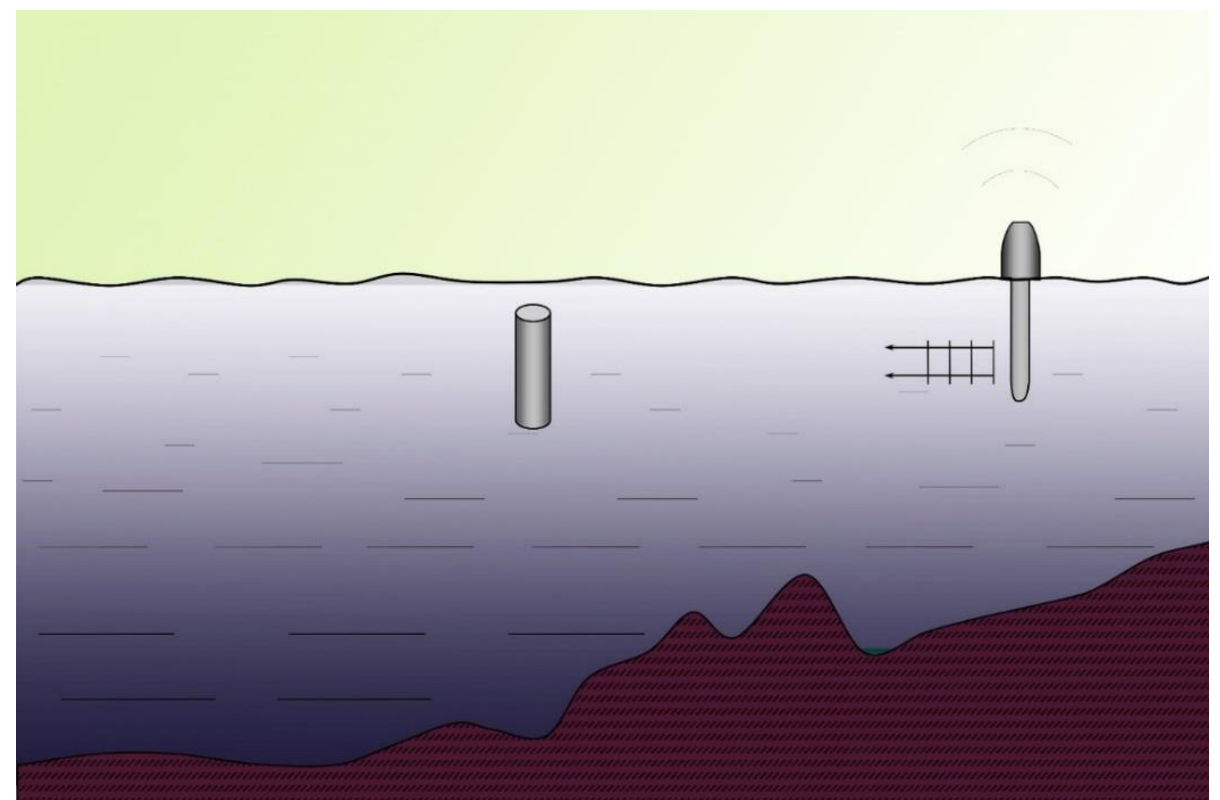

Figure1. Hydroacoustic system for assessing the underwater environment of shallow water

\section{Statement of the problem of scattering by a cylinder}

We will consider wave processes when a cylindrical scatterer is located in the region of nonlinear interaction of primary pump waves of an acoustic parametric antenna (Abbasov, 2016). In this case, the condition of the planewave incidence of pump waves onto the cylinder must be observed, therefore, the dimensions of the parametric antenna should be an order of magnitude larger than the radius of the cylindrical body. Then the cylindrical scatterer will be located in the near zone of the parametric antenna, where the incident wave has a flat shape. In our case, the cylindrical diffuser is acoustically rigid and satisfies the Neumann boundary condition. It should be noted that the pump waves of the parametric antenna are high-frequency, therefore, the scattering for them is geometric. For this reason, we consider the scattering problem in the high-frequency asymptotic limit.

The geometry of the problem for the three-dimensional case is shown in Fig. 2, the axis of the cylinder of infinite length coincides with the axis $Z$ of the cylindrical coordinate system. A plane wave is an incident on a cylindrical surface parallel to the $Z$ axis. As a result of the scattering of a plane wave on a cylindrical surface, a scattered cylindrical wave will propagate in the surrounding space. In this case, the total primary acoustic pressure field will be determined by the expression: 


$$
p^{(1)}=p_{n i}+p_{n s} \text {, }
$$

where $p_{n i}$ the acoustic pressure of the incident and $p_{n s}$ - scattered waves, $\mathrm{n}=1,2$ corresponds to the incident waves with frequencies $\omega 1$ and $\omega 2$.

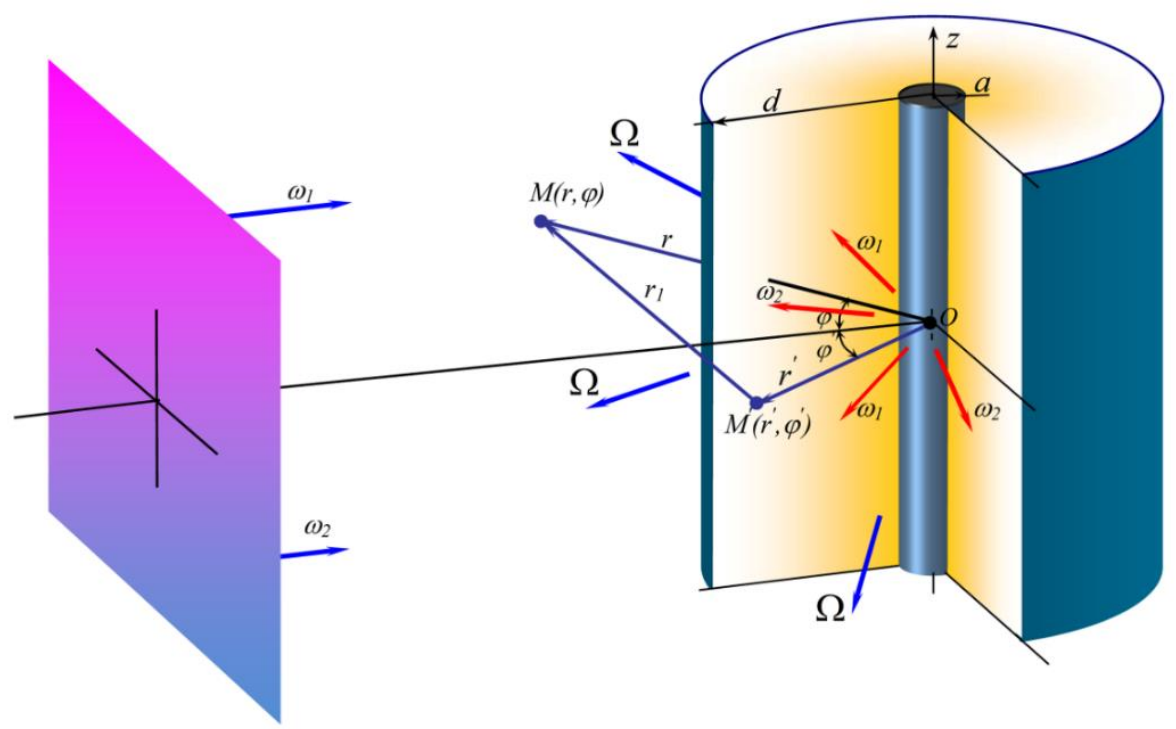

Figure 2. The geometry of the scattering problem in three-dimensional space

Wave phenomena for this case are described by a nonlinear wave equation (Novikov et al., 1987):

$$
\Delta p-\frac{1}{c_{0}^{2}} \frac{\partial^{2} p}{\partial t^{2}}+\frac{b}{c_{0}^{2} \rho_{0}} \frac{\partial}{\partial t} \Delta p=-Q,
$$

where $Q(p, \vartheta)=\frac{1}{c_{0}^{4} \rho_{0}}\left(\frac{\partial p}{\partial t}\right)^{2}+\frac{(\varepsilon-1)}{c_{0}^{4} \rho_{0}} \frac{\partial^{2} p^{2}}{\partial t^{2}}+\frac{\rho_{0}}{2} \Delta \mathbf{v}^{2}+\rho_{0} \mathbf{v} \nabla \nabla \mathbf{v}$ is a group of nonlinear terms, $c_{0}$ the speed of sound in an aqueous medium, $\rho_{0}$ the density of an unperturbed medium, $\varepsilon$ - is the parameter of quadratic nonlinearity, $b$ is the dissipative coefficient of the medium, $\mathbf{V}$ - is the vibrational velocity.

To solve the wave equation (2), we will use the method of successive approximations of series expansion in a small parameter, in this case, the small parameter is the Mach number:

$$
p=M p^{(1)}+M^{2} p^{(2)},
$$

it is considered, that the following conditions are met $\left|\frac{p^{(n+1)}}{p^{(n)}}\right| \approx M, M=\frac{\vartheta_{0}}{c_{0}}=\frac{\xi_{0}}{\lambda}<<1$, this inequality is fulfilled in water up to radiation levels of $280 \mathrm{~dB}$ relative to $1 \mu \mathrm{Pa}$.

According to the small parameter method, to a first approximation, nonlinear terms in equation (2) are not taken into account, it is assumed that $Q=0$. This corresponds to the linear problem of wave scattering in a dissipative medium, therefore, the solution $p^{(1)}$ to the first approximation problem will be an expression (1). Then the spectral components of the function $Q$ are determined at the frequencies of the secondary field waves. To find the acoustic pressure of the waves of the secondary field $p^{(2)}$ in the second approximation, the following linear inhomogeneous wave equation is solved:

Abbasov, I. (2019). A research and modeling of wave processes at the scattering of nonlinear acoustic waves on cylindrical bodies. International Research Journal of Engineering, IT \& Scientific Research, 5(5), 32-44. https://doi.org/10.21744/irjeis.v5n5.779 


$$
\Delta p^{(2)}-\frac{1}{c_{0}^{2}} \frac{\partial^{2} p^{(2)}}{\partial t^{2}}=-Q=-\frac{\varepsilon}{c_{0}^{4} \rho_{0}} \frac{\partial^{2} p^{(1)^{2}}}{\partial t^{2}}
$$

\section{The solution of a nonlinear wave equation for a different frequency wave}

Further, equation (3) is transformed into the inhomogeneous Helmholtz equation, its solution is the volume integral, which consists of the product of the Green's function and the density of sources of secondary waves of difference frequency (Novikov et al., 1987); (Dean, 1962):

$$
P_{-}^{(2)}(r, \varphi, z)=\frac{1}{4 \pi} \int_{V} q_{-}\left(r^{\prime}, \varphi^{\prime}, z^{\prime}\right) G\left(r_{1}\right) r^{\prime} d \varphi^{\prime} d z^{\prime} d r^{\prime}
$$

where $G\left(r_{1}\right)=\exp \left(-i k_{-} r_{1}\right) / r_{1}$ - Green's function, $r^{\prime}, \varphi^{\prime}, z^{\prime}$ - coordinates of the current volume point, $M^{\prime}\left(r^{\prime}, \varphi^{\prime}, z^{\prime}\right), r$-distance to the observation point, $M(r, \varphi, z), r_{1}$ - the distance between the current volume point $M^{\prime}\left(r^{\prime}, \varphi^{\prime}, z^{\prime}\right)$ and the observation point $M(r, \varphi, z)$ (Fig. 2).

It is necessary to determine the Green's function for our case, the distance $r_{1}$ in the Cartesian coordinate system will be determined as

$$
r_{1}=\sqrt{\left(x-x^{\prime}\right)^{2}+\left(y-y^{\prime}\right)^{2}+\left(z-z^{\prime}\right)^{2}}
$$

If we take into account the known relations between Cartesian $(x, y, z)$ and cylindrical coordinates $(r, \varphi, z)$ (Korn \& Korn, 1961), we obtain

$$
x=r \cos \varphi, \quad y=r \sin \varphi, \quad z=z .
$$

In this case, in the far-field $r^{\prime}<<r, r_{1}$ expression (5) is converted to the form

$$
r_{1}=\sqrt{\left(r \cos \varphi-r^{\prime} \cos \varphi^{\prime}\right)^{2}+\left(r \sin \varphi-r^{\prime} \sin \varphi^{\prime}\right)^{2}} \approx r \sqrt{1-2 \frac{r^{\prime}}{r} \cos \left(\varphi-\varphi^{\prime}\right)}
$$

Next, we expand expression (6) in a row with precision to the second term [Dwight, 1983]

$$
r_{1} \approx r\left(1-\frac{1}{2}\left(2 \frac{r^{\prime}}{r} \cos \left(\varphi-\varphi^{\prime}\right)\right)\right)=r-r^{\prime} \cos \left(\varphi-\varphi^{\prime}\right)
$$

We substitute the obtained expressions into the original expression for the Green's function; this function in the farfield will be determined by the following asymptotic expression:

$$
G\left(r_{1}\right)=\exp \left(-i k_{-}\left(r-r^{\prime} \cos \left(\varphi-\varphi^{\prime}\right)\right) / r\right.
$$

Integration in expression (4) is carried out over the volume $V$, which is filled with sources of secondary waves and limited by the cylindrical coordinates by the relations:

$$
a \leq r^{\prime} \leq d, \quad 0 \leq \varphi^{\prime} \leq \pi,-z_{\Lambda} \leq z^{\prime} \leq z_{\Lambda}
$$

where $z_{\Lambda} \gg \Lambda, \Lambda$ - wavelength of the differential frequency, the volume $V$ is a cylindrical layer of the medium around the diffuser with an internal radius $a$ (radius of the cylinder) and external $d$ (Fig. 2). The distance $d$ is determined by the length of the region of nonlinear interaction of high-frequency pump waves; we consider that the source waves are almost completely attenuated behind this region.

Given the expression for the Green's function (7), expression (4) is transformed to

$$
P_{-}^{(2)}(r, \varphi, z)=\frac{\exp \left(-i k_{-} r\right)}{4 \pi r} \int_{a}^{d} \int_{0}^{\pi} \int_{-z}^{z} q_{-}\left(r^{\prime}, \varphi^{\prime}, z^{\prime}\right) \exp \left(i k_{-} r^{\prime} \cos \left(\varphi-\varphi^{\prime}\right)\right) r^{\prime} d \varphi^{\prime} d z^{\prime} d r^{\prime}
$$


We integrate expression (8), since it does not depend on the coordinate $z$, we consider separately the integral over the coordinate $\varphi^{\prime}$ (with replacement $\alpha=k_{-} r^{\prime}, \beta=\varphi-\varphi^{\prime}$ )

$$
\int_{0}^{\pi} \exp \left(i k_{-} r \cos \left(\varphi-\varphi^{\prime}\right)\right) d \varphi^{\prime}=-\int_{\varphi}^{\varphi-\pi} \cos (\alpha \cos \beta) d \beta-i \int_{\varphi}^{\varphi-\pi} \sin (\alpha \cos \beta) d \beta
$$

The first part of this expression, taking into account the expansion in a series of cylindrical functions (Dwight, 1983), takes the form

$$
\int_{\varphi}^{\varphi-\pi} \cos (\alpha \cos \beta) d \beta=\pi J_{0}(\alpha)
$$

The second part of the expression after expansion in a row up to the second term will have the form

$$
\int_{\varphi}^{\varphi-\pi} \sin (\alpha \cos \beta) d \beta \approx-4 J_{1}(\alpha) \sin \varphi+\frac{4}{3} J_{3}(\alpha) \sin 3 \varphi
$$

The initial expression (9), taking into account the obtained solutions, will take the form

$$
\int_{0}^{\pi} \exp \left(i k_{-} r \cos \left(\varphi-\varphi^{\prime}\right)\right) d \varphi^{\prime}=-\pi J_{0}\left(k_{-} r^{\prime}\right)+4 i J_{1}\left(k_{-} r^{\prime}\right) \sin \varphi-i \frac{4}{3} J_{3}\left(k_{-} r^{\prime}\right) \sin 3 \varphi
$$

Next, we take into account the asymptotic row expansion for the cylindrical Bessel function (Skudrzyk, 1971)

$$
J_{m}(x)=\sqrt{\frac{2}{\pi x}} \cos \left(x-\frac{2 m+1}{4} \pi\right)
$$

then expression (10) is transformed to

$$
\int_{0}^{\pi} \exp \left(i k_{-} r \cos \left(\varphi-\varphi^{\prime}\right)\right) d \varphi^{\prime} \approx-\pi \sqrt{\frac{2}{\pi k_{-} r^{\prime}}} \cos k_{-} r^{\prime}(1+i \sin \varphi) .
$$

The volume integral of expression (8), taking into account solution (11), will take the form

$$
P_{-}^{(2)}(r, \varphi, z)=-\frac{\exp \left(-i k_{-} r\right)(1+i \sin \varphi)}{2 r \sqrt{2 \pi k_{-}}} \int_{a}^{d} q_{-}\left(r^{\prime}\right) \cos k_{-} r^{\prime} \sqrt{r^{\prime}} d r^{\prime} \text {. }
$$

Taking into account integration with respect to the coordinates $z^{\prime}$ and $\varphi^{\prime}$, the expression for the function of the sources of the secondary waves $Q$, in the second approximation we obtain the following expression for the total acoustic pressure of the difference-frequency wave:

$$
P_{-}^{(2)}(r, \varphi, z)=P_{-1}^{(2)}(r, \varphi, z)+P_{-2}^{(2)}(r, \varphi, z)+P_{-3}^{(2)}(r, \varphi, z)+P_{-4}^{(2)}(r, \varphi, z)
$$

This expression for the total acoustic pressure of a difference-frequency wave $P_{-}^{(2)}(r, \varphi, z)$ consists of four spatial terms. The first term corresponds to the nonlinear interaction of incident plane high-frequency waves $\omega_{1}$ and $\omega_{2}$. The second term describes the interaction of an incident plane frequency wave $\omega_{1}$ with a scattered cylindrical frequency wave $\omega_{2}$. The third term corresponds to the interaction of the incident plane $\omega_{2}$ wave with the scattered cylindrical $\omega_{1}$, and the fourth term characterizes the interaction of scattered cylindrical waves with frequencies $\omega_{1}$ and $\omega_{2}$. It can be noted that, within the framework of the considered wave processes, a nonlinear interaction occurs between acoustic waves with different spatial configurations of the wavefront.

Abbasov, I. (2019). A research and modeling of wave processes at the scattering of nonlinear acoustic waves on cylindrical bodies. International Research Journal of Engineering, IT \& Scientific Research, 5(5), 32-44. https://doi.org/10.21744/irjeis.v5n5.779 


\section{Construction of scattering diagrams}

Further, for the calculation and construction hard hat scattering diagram acoustic pressure program «CylScatt» was developed. Figure 3 shows the scheme of the algorithm of this program, which consists of the following steps:

a) Input data is set: linear frequencies of the initial incident waves $f_{1}, f_{2}$, the speed of sound in the aquatic environment, $c$, water density $\rho$, cylinder radius $a$, nonlinear parameter for the aquatic environment $\varepsilon$; the amplitude of the acoustic wave $A$, the initial and final value of the angle interval is $\varphi-0^{\circ} \div 180^{\circ}$, angle $\operatorname{step} \varphi-0,2^{0}$.

b) The wave parameters of the cylindrical diffuser are calculated: linear frequencies of the difference frequency wave $\left(F_{-}\right)$, wavelength of initial waves and difference frequency $\left(\lambda_{1} ; \lambda_{2} ; \Lambda_{-}\right)$, wave numbers of initial waves and difference frequency $\left(k_{1} ; k_{2} ; k_{-}\right)$, the length of the region of nonlinear interaction of the initial high-frequency waves $d$, wave dimensions of the cylinder on the initial and secondary wave $\left(k_{1} a ; k_{2} a\right.$; $k_{-} a$ ), calculation of the components of the spatial term of the acoustic pressure of the differential frequency wave $\left(P_{-n}^{(2)}(r, \varphi, z)\right.$.

c) Calculation of the total acoustic pressure of the differential frequency wave $\left(P_{-}^{(2)}(r, \varphi, z)\right.$.

d) Construction the scattering diagram of the total acoustic wave of difference frequency $\left(P_{-}^{(2)}(r, \varphi, z)\right.$.

e) Construction a three-dimensional diagram of the scattering on the cylinder of a wave of different frequency.

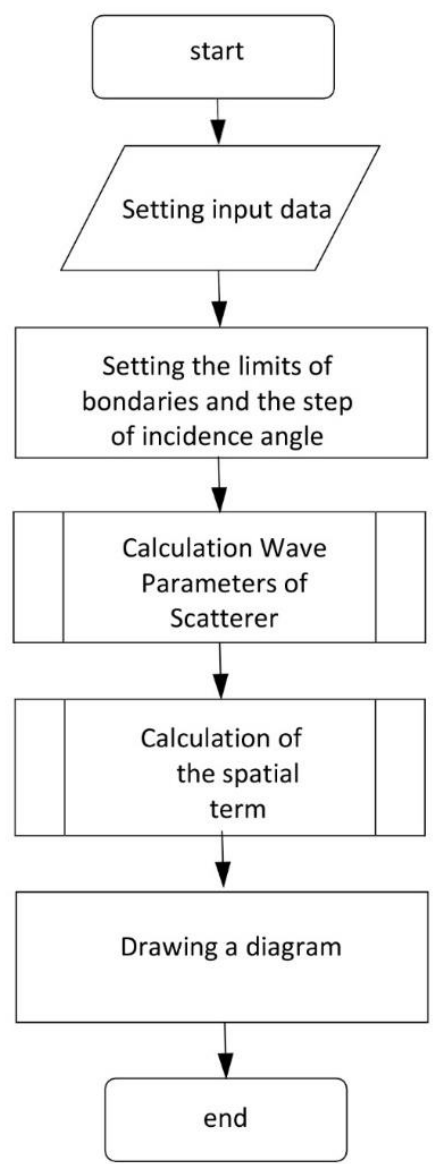

Figure 3. Scheme the algorithm of the program «CylScatt» 
In Fig.4. are presented scattering diagrams of the total acoustic pressure of difference frequency wave $P_{-}^{(2)}(r, \varphi, z)$ on the cylinder with radius $a=0.01 \mathrm{~m}$ at constant wave dimension $k_{-} a=5$ a distance from the cylinder $d=0.035 \mathrm{~m}$ (primary wave frequencies $f_{2}=1000 \mathrm{kHz}, f_{1}=880 \mathrm{kHz}$, difference wave frequency $F_{-}=120$ $\mathrm{kHz})$.

Analyzing the scattering diagram, it can note that while an increase in the length of the cylindrical layer around the scatterer leads to some narrowing of the main maxima. The latter property is characteristic of parametric antennas by analogy with an increase in the dimensions of the reradiation region. The primary plane waves form a scattering field in the forward and backward directions, and the scattered cylindrical waves create scattering in the lateral directions.

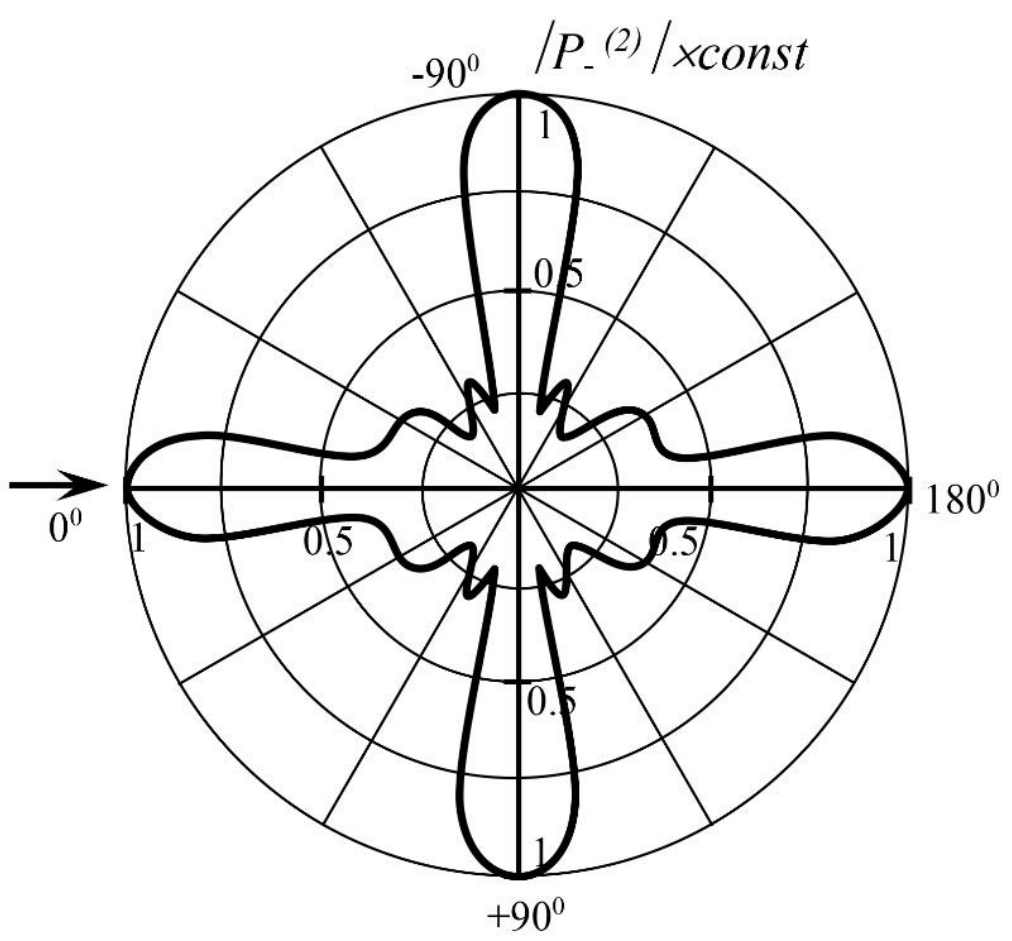

Figure 4. Scattering diagrams of the total acoustic pressure of difference frequency wave $P_{-}^{(2)}(r, \varphi, z)$ on the cylinder with radius $a=0.01 \mathrm{~m}$ at constant wave dimension $k_{-} a=5$ a distance from the cylinder $d=0.035 \mathrm{~m}$

Figure 5 shows a three-dimensional model of the scattering diagram of the total acoustic pressure of a difference frequency wave $P_{-}^{(2)}(r, \varphi, z)$ on a rigid cylinder. A three-dimensional model in the form of an extruded body with a cutout of the fourth part is shown in a rectangular geometry. It clearly shows the distribution of the acoustic field around the cylindrical scatterer. On the horizontal plane xoy there is a curve of the scattering diagram shown in Fig. 4.

The scattering diagram has the main lobes in the directions $\varphi=0^{0}, \pm \pi / 2$ and $\pi$, these directions correspond to the directions of the minimum phase differences of the nonlinearly interacting initial high-frequency waves. Incident plane waves form a scattering field in the opposite and forward directions, and scattered cylindrical waves in the lateral directions (Abbasov \& Zagrai, 2017).

Abbasov, I. (2019). A research and modeling of wave processes at the scattering of nonlinear acoustic waves on cylindrical bodies. International Research Journal of Engineering, IT \& Scientific Research, 5(5), 32-44. https://doi.org/10.21744/irjeis.v5n5.779 


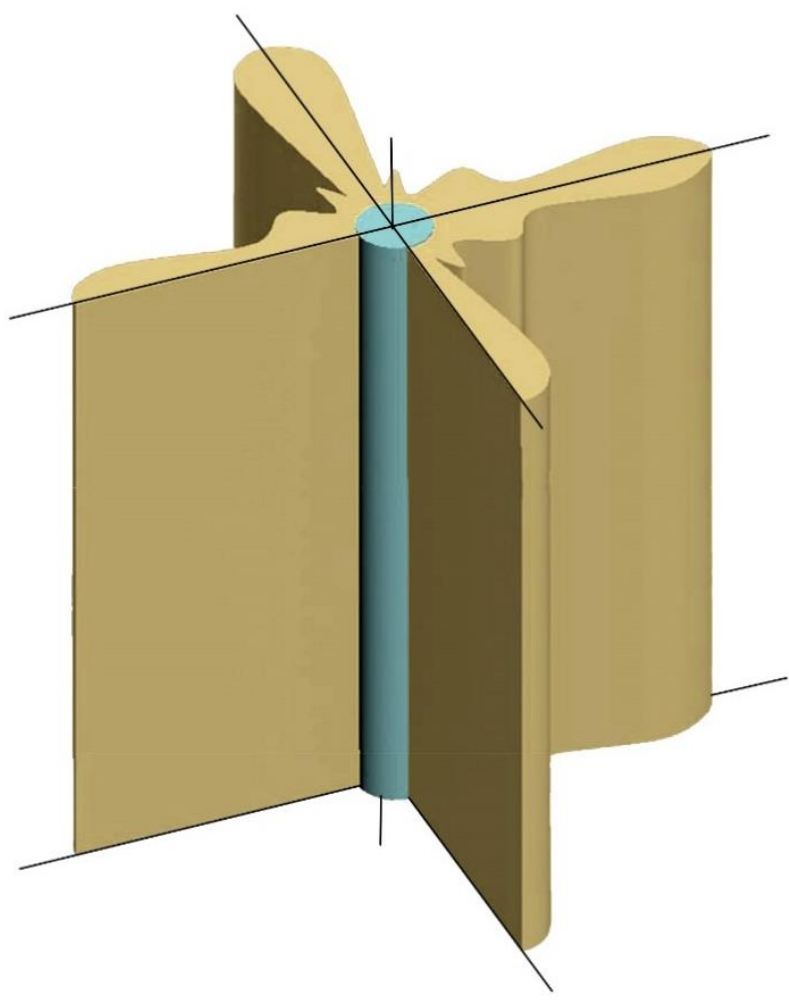

Figure 5. The three-dimensional model of the scattering diagram the total acoustic pressure difference frequency wave $P_{-}^{(2)}(r, \varphi, z)$ on a cylinder with a radius $a=0.01 \mathrm{~m}$ at $k_{-} a=5, d=0.035 \mathrm{~m}$

\section{Determination of the radius of the circle of convergence}

We used the small parameter method to solve the nonlinear wave equation (2), it is necessary to determine the limits of applicability of this method, i.e. radius of convergence for a given decomposition. In a second approximation, acoustic pressure $p^{(2)}$ is a solution to a linear inhomogeneous wave equation consisting of four spectral components of the secondary field $2 \omega_{1}, \omega_{2}-\omega_{1}=\Omega, \omega_{2}+\omega_{1}$ and $2 \omega_{2}$ :

$$
p^{(2)}=p_{2 \omega_{1}}^{(2)}+p_{-}^{(2)}+p_{+}^{(2)}+p_{2 \omega 2}^{(2)} \text {. }
$$

We write out the amplitude parts of the acoustic pressure for each spectral component, for the second-harmonic $2 \omega_{1}$ acoustic pressure, it has the form:

$$
P_{0 \omega_{1}}^{(2)} \approx \frac{\varepsilon \Psi_{10}^{2} \omega_{1}^{2} \rho_{0}}{2 c_{0}^{4} k_{1} r \sqrt{\pi k_{1}}}+\frac{\varepsilon \Psi_{10}^{2} \omega_{1}^{2} \rho_{0} A_{0}}{c_{0}^{4} k_{1}^{2} r \pi}+\frac{\varepsilon \Psi_{10}^{2} \omega_{1}^{2} \rho_{0} A_{0}^{2}}{2 c_{0}^{4} \pi k_{1} r \sqrt{\pi k_{1}}}
$$

If we consider that $k_{1}, k_{2}>>1$ and reflection coefficient $A_{0}<1$ :

$$
P_{02 \omega_{1}}^{(2)} \approx \frac{\Psi_{10}^{2} \varepsilon \omega_{1}^{2} \rho_{0}}{2 c_{0}^{4} k_{1} r \sqrt{\pi k_{1}}}
$$

For the amplitude part of the acoustic pressure of the second harmonic wave $2 \omega_{2}$, we similarly obtain: 


$$
P_{0}^{(2)} \approx \frac{\Psi_{20}^{2} \varepsilon \omega_{2}^{2} \rho_{0}}{2 c_{0}^{4} k_{2} r \sqrt{\pi k_{2}}} .
$$

The expression for the amplitude part of the acoustic pressure of the differential frequency wave will have the form $\omega_{2}-\omega_{1}=\Omega$ :

$$
P_{0}^{(2)} \approx \frac{4 \Psi_{10}^{2} \varepsilon \omega_{1} \Omega^{2} \rho_{0}}{c_{0}^{4} r k_{-} \sqrt{2 \pi k_{-}}}+\frac{4 \Psi_{10}^{2} \varepsilon \omega_{1} \Omega^{2} \rho_{0} A_{0}}{c_{0}^{4} r \pi k_{1} \sqrt{k_{-} k_{2}}}+\frac{4 \Psi_{10}^{2} \varepsilon \omega_{1} \Omega^{2} \rho_{0} A_{0}^{2}}{c_{0}^{4} r \pi \sqrt{2 \pi k_{1} k_{2} k_{-}}}
$$

If we consider that $k_{1}, k_{2}>>1, k_{-}<k_{1}, k_{2}$ and reflection coefficient $A_{0}<1$ :

$$
P_{0}^{(2)} \approx \frac{4 \Psi_{10}^{2} \varepsilon \omega_{1} \Omega^{2} \rho_{0}}{c_{0}^{4} r k_{-} \sqrt{2 \pi k_{-}}} \text {. }
$$

Similarly, the amplitude part of the acoustic pressure of the wave of the total frequency $\omega_{2}+\omega_{1}$ will have the form:

$$
P_{0}^{(2)} \approx \frac{4 \Psi_{10}^{2} \varepsilon \omega_{1}\left(2 \omega_{1}\right)^{2} \rho_{0}}{c_{0}^{4} r k_{+} \sqrt{2 \pi k_{+}}} \approx \frac{16 \Psi_{10}^{2} \varepsilon \omega_{1}^{3} \rho_{0}}{c_{0}^{4} r k_{+} \sqrt{2 \pi k_{+}}}
$$

We substitute the amplitude parts of the acoustic pressure into the expression for the solution in the second approximation (12):

$$
P_{0}^{(2)} \approx \frac{\Psi_{10}^{2} \varepsilon \omega_{1}^{2} \rho_{0}}{2 c_{0}^{4} k_{1} r \sqrt{\pi k_{1}}}+\frac{4 \Psi_{10}^{2} \varepsilon \omega_{1} \Omega^{2} \rho_{0}}{c_{0}^{4} r k_{-} \sqrt{2 \pi k_{-}}}+\frac{16 \Psi_{10}^{2} \varepsilon \omega_{1}^{3} \rho_{0}}{c_{0}^{4} r k_{+} \sqrt{2 \pi k_{+}}}+\frac{\Psi_{10}^{2} \varepsilon \omega_{2}^{2} \rho_{0}}{2 c_{0}^{4} k_{2} r \sqrt{\pi k_{2}}} .
$$

The convergence distance of the decomposition method is determined by the value of the characteristic time $t_{c h a r}$, which is determined by equating the terms of a number of different approximations:

$$
M p_{0}^{(1)}\left(r, \varphi, z, t_{\text {char },}\right)=M^{2} p_{0}^{(2)}\left(r, \varphi, z, t_{\text {char. }}\right) .
$$

The characteristic time $t_{\text {char. }}$ is determined by the radius of the circle of convergence of the expansion in the small parameter $M$. To obtain this parameter, we equate the amplitude coefficients of the terms of the first and second approximations:

$$
\begin{gathered}
M 2 \Psi_{10} \omega_{1} \rho_{0} \varepsilon \sqrt{\frac{2}{\pi k_{1} r}}=M^{2}\left(\frac{\Psi_{10}^{2} \varepsilon \omega_{1}^{2} \rho_{0}}{2 c_{0}^{4} k_{1} r \sqrt{\pi k_{1}}}+\frac{4 \Psi_{10}^{2} \delta \omega_{1} \Omega^{2} \rho_{0}}{c_{0}^{4} r k_{-} \sqrt{2 \pi k_{-}}}+\frac{16 \Psi_{10}^{2} \varepsilon \omega_{1}^{3} \rho_{0}}{c_{0}^{4} r k_{+} \sqrt{2 \pi k_{+}}}+\frac{\varepsilon \Psi_{10}^{2} \omega_{2}^{2} \rho_{0}}{2 c_{0}^{4} k_{2} r \sqrt{\pi k_{2}}}\right) \cdot t_{c h a r}^{2} \\
t_{c a r .}^{2}=\frac{c_{0}^{4} k_{1} \sqrt{2 r}}{10 M \Psi_{10} \omega_{1}^{2}}=\frac{c_{0}^{2} k_{1} \sqrt{2 r}}{10 M \Psi_{10}\left(\omega_{1}^{2} / c_{0}^{2}\right)}=\frac{c_{0}^{2} k_{1} \sqrt{2 r}}{10 M \Psi_{10} k_{1}^{2}}=\frac{c_{0}^{2} \sqrt{2 r}}{10 M \Psi_{10} k_{1}} \\
t_{\text {char. }} \approx \sqrt{\frac{c_{0}^{2} \sqrt{r}}{2 M \Psi_{10} k_{1}}}
\end{gathered}
$$

This expression (19) for a characteristic time allows one to determine the radius of the circle of convergence of the expansion in the small parameter $M$. Outside the value of the radius of convergence, the expansion in a series in the small parameter $M$ becomes divergent, and the research task loses its physical meaning.

Abbasov, I. (2019). A research and modeling of wave processes at the scattering of nonlinear acoustic waves on cylindrical bodies. International Research Journal of Engineering, IT \& Scientific Research, 5(5), 32-44. 


\section{Conclusion}

In conclusion, it can be noted that the article investigated the process of scattering of nonlinear acoustic waves on cylindrical bodies, describes the features of the applied methods and wave processes. A hydroacoustic system is proposed for assessing the underwater situation in shallow water areas. Using the method of successive approximations, we obtained the asymptotic expression for the acoustic pressure of a difference frequency wave. Using the developed program, scattering diagrams were calculated, three-dimensional models of the problem statement and scattering diagrams on a cylinder are presented. The radius of convergence of the used method of expansion in a small parameter was also determined.

\section{Conflict of interest statement}

The author declared that he have no competing interests.

\section{Statement of authorship}

The author have a responsibility for the conception and design of the study. The author have approved the final article.

\section{Acknowledgments}

The authors would like to thank the reviewer for their consideration of the further process of the present paper. Thanks to the editor of IRJEIS for the valuable support, time as well as advice. 


\section{References}

Abbasov, I. B. (Ed.). (2016). Hyrdoacoustic Ocean Exploration: Theories and Experimental Application. John Wiley \& Sons.

Abbasov, I. B., \& Zagrai, N. P. (2019). Wave Problems of Scattering on Cylindrical Bodies. In 2019 Radiation and Scattering of Electromagnetic Waves (RSEMW) (pp. 228-231). IEEE. https://doi.org/10.1109/RSEMW.2019.8792694

Abbasov, I. B., \& Zagrai, N. P. (2017). The problems of wave scattering by spheroidal bodies. In 2017 Radiation and Scattering of Electromagnetic Waves (RSEMW) (pp. 84-86). IEEE. https://doi.org/10.1109/RSEMW.2017.8103571

Andronov, I. V., \& Lavrov, Y. A. (2015). Scattering by an elliptic cylinder with a strongly elongated cross section. Acoustical Physics, 61(4), 383-387. https://doi.org/10.1134/S1063771015040016

Dean III, L. W. (1962). Interactions between sound waves. The Journal of the Acoustical Society of America, 34(8), 1039-1044. https://doi.org/10.1121/1.1918241

Desjouy, C., Ollivier, S., Marsden, O., Karzova, M., \& Blanc-Benon, P. (2016). Irregular reflection of weak acoustic shock pulses on rigid boundaries: Schlieren experiments and direct numerical simulation based on a NavierStokes solver. Physics of Fluids, 28(2), 027102. https://doi.org/10.1063/1.4940987

Dmitriev, K. V. (2018). Scattering of an Acoustic Field by Refraction-Density Inhomogeneities with a Small Wave Size and Solution of the Problem of Direct Scattering in an Inhomogeneous Medium. Acoustical Physics, 64(2), 131-143. https://doi.org/10.1134/S1063771018020033

Dos Santos, S., Lints, M., Poirot, N., \& Salupere, A. (2015). Optimized excitation for nonlinear wave propagation in complex media: From biomedical acoustic imaging to nondestructive testing of cultural heritage. The Journal of the Acoustical Society of America, 138(3), 1796-1796. https://doi.org/10.1121/1.4933698

Duck, F. A. (2002). Nonlinear acoustics in diagnostic ultrasound. Ultrasound in medicine \& biology, 28(1), 1-18. https://doi.org/10.1016/S0301-5629(01)00463-X

Dwight, G. B. (1983). Tablitsy integralov i drugie matematicheskie formuly [Tables of Integrals and Other Mathematical Formulas].

Gurbatov, S. N., Gryaznova, I. Y., \& Ivashchenko, E. N. (2016). Study of acoustic wave backscattering by discrete inhomogeneities of different $\quad$ sizes. Acoustical Physics, 62(2), 202-206. https://doi.org/10.1134/S106377101602007X

Kleshchev, A. A. (2011). Scattering of low-frequency pulsed sound signals from elastic cylindrical shells. Acoustical Physics, 57(3), 375. https://doi.org/10.1134/S1063771011020102

Korn, G. A., \& Korn, T. M. (2000). Mathematical handbook for scientists and engineers: definitions, theorems, and formulas for reference and review. Courier Corporation.

Larin, N. V., \& Tolokonnikov, L. A. (2015). The scattering of a plane sound wave by an elastic cylinder with a discrete-layered covering. Journal of Applied Mathematics and Mechanics, 79(2), 164-169. https://doi.org/10.1016/j.jappmathmech.2015.07.007

Mitri, F. G. (2016). Acoustic backscattering and radiation force on a rigid elliptical cylinder in plane progressive waves. Ultrasonics, 66, 27-33. https://doi.org/10.1016/j.ultras.2015.12.003

Novikov, B. K., Rudenko, O. V., Timoshenko, V. I., \& Breazeale, M. A. (1988). Nonlinear Underwater Acoustics by BK Novikov, OV Rudenko, and VI Timoshenko. https://doi.org/10.1121/1.396924

Ostashev, V. E., \& Wilson, D. K. (2015). Acoustics in moving inhomogeneous media. CRC Press.

Partridge, C., \& Smith, E. R. (1995). Acoustic scattering from bodies: Range of validity of the deformed cylinder method. The Journal of the Acoustical Society of America, 97(2), 784-795. https://doi.org/10.1121/1.412943

Rosa-Santos, P., Taveira-Pinto, F., Teixeira, L., \& Ribeiro, J. (2015). CECO wave energy converter: Experimental proof of concept. Journal of Renewable and Sustainable Energy, 7(6), 061704. https://doi.org/10.1063/1.4938179

Skudrzyk, Eugen. The foundations of acoustics: basic mathematics and basic acoustics. Springer Science \& Business Media, 2012.

Abbasov, I. (2019). A research and modeling of wave processes at the scattering of nonlinear acoustic waves on cylindrical bodies. International Research Journal of Engineering, IT \& Scientific Research, 5(5), 32-44. https://doi.org/10.21744/irjeis.v5n5.779 


\section{Biography of Author}

\begin{tabular}{|l|l||}
\hline \hline & $\begin{array}{l}\text { Prof. Iftikhar B. Abbasov is Head of Department of Engineering Graphics and } \\
\text { Computer Design, Engineering-Technological Academy of Southern Federal } \\
\text { University, Taganrog, Russia. He is the author of } 13 \text { monographs and } 14 \text { textbooks. } \\
\text { The scientific area: mathematical simulation of nonlinear wave phenomena in fluid } \\
\text { dynamics and acoustics, computer modeling in industrial design. } \\
\text { Email: iftikhar_abbasov@mail.ru }\end{array}$ \\
\hline
\end{tabular}

